\title{
A Statistical Model of Magnetic Islands in a Large Current Layer
}

\author{
R. L. Fermo, J. F. Drake, and M. Swisdak \\ IREAP, University of Maryland, College Park, MD 20742-3511, USA
}

(Dated: September 6, 2021)

\begin{abstract}
We develop a statistical model describing the dynamics of magnetic islands in very large current layers that develop in space plasma. Two parameters characterize the island distribution: the flux $\psi$ contained in the island and the area $A$ it encloses. We derive an integro-differential evolution equation for this distribution function, based on rules that govern the small-scale generation of secondary islands, the rates of island growth, and island merging. Our numerical solutions of this equation produce island distributions relevant to the magnetosphere and corona. We also derive and analytically solve a differential equation for large islands that explicitly shows the role merging plays in island growth.
\end{abstract}

Recent reconnection simulations have shown that guide field reconnection becomes bursty [1]. Electron current layers near the magnetic x-line lengthen and become unstable to the formation of secondary magnetic islands, which dynamically grow in size and begin to merge. The existence of these magnetic islands is consistent with observations in both the magnetotail and the magnetopause [2], and their occurrence in current layers on the sun has also been inferred [3]. The presence of many islands could also account for the observed thickness of current sheets associated with coronal mass ejections (CMEs), which is far greater than that predicted by reconnection models employing classical or anomalous resistivity [4].

Magnetic island formation is of great interest because of its impact on reconnection rates and because both simulations [5] and observations [6] indicate that islands are correlated with highly energetic electrons. However, the dynamics of islands in very large-scale current layers are not yet well-understood. On the one hand, even the largest particle-in-cell (PIC) simulations [7] come nowhere near the scale sizes of these current layers $\left(L \sim 4000 d_{i}\right.$ in the magnetopause, $L \sim 10^{6} d_{i}$ in the corona). On the other hand, fluid simulations that encompass these large scales [8] fail to capture the smallscale dynamics of reconnection within the dissipation region.

In light of these limitations, we employ a novel statistical method for treating two-dimensional magnetic islands in large current layers. We define a statistical distrubtion function which describes islands in the whole current layer and develop an evolution equation for that distribution. We then present steady-state solutions that show how the merger of smaller islands drives the growth of large islands. The model is based on rules of island merging based on two-dimensional particle-in-cell reconnection simulations.

We model the islands using a distribution function $f$ in terms of the island's flux $\psi$ and area $A$. Defined in this manner, $d N=f(\psi, A) d \psi d A$ signifies the number of magnetic islands with flux in the range $[\psi, \psi+d \psi]$ and area in $[A, A+d A]$. The distribution function $f(\psi, A, t)$ evolves in time in the phase space of $(\psi, A)$, independent of a space coordinate, because it describes islands over the whole current sheet of length $L$. The two quantities $\psi$ and $A$ are sufficient to completely characterize the state of an island: the magnetic field strength is computed as $B=\psi / r=\psi \sqrt{\pi / A}$, where $r=\sqrt{A / \pi}$ is a characteristic radius.

With the distribution function defined, we now establish rules for the behavior of magnetic islands, and formulate how these rules affect the distribution. Islands first form at scales between the electron $d_{e}$ and ion $d_{i}$ skin depths in the current layers near x-lines due to the tearing instability [1]. The evolution equation therefore includes a source term $S(\psi, A)$ at this scale, in our case of Gaussian shape. As islands convect outwards at the Alfvén speed, a sink term models the convection of islands out of the system at the rate $c_{A} / L$.

Furthermore, the model must account for the growth of magnetic islands due to reconnection. PIC simulations have shown that once reconnection reaches a nonlinear stage, the normalized reconnection rate plateaus at roughly $\varepsilon \approx 0.1[9]$. Given this constant reconnection rate and an asymptotic reconnection magnetic field of $B_{0}$, the magnetic flux of an island increases at the rate $\dot{\psi}=v_{i n} B_{x}=\varepsilon c_{A} B_{0}$. Likewise, the island's characteristic radius $r$ increases at a constant rate $\dot{r}=\varepsilon c_{A}$, and so the area increases at the rate $\dot{A}=2 \pi r v_{i n}=2 \varepsilon c_{A} \sqrt{\pi A}$.

We now describe the rules for island merger: the merger of two islands yields an island with an area $A$ equal to the sum of the two initial areas and a flux $\psi$ equal to the higher of the two initial fluxes. The flux does not add because magnetic reconnection does not increase or decrease magnetic flux but simply changes its connectivity. Thus, field lines from the island with more flux reconnect with those from the island with less flux until all of the latter's flux is depleted. The simplicity of these rules reflect our choice of $\psi$ and $A$ as the variables defining our phase space. Fig. 1 shows results from a PIC simulation that demonstrates this in the simple case of two isolated flux bundles. Magnetic field contours are shown at various times during the merging process, with the outermost field line representing the boundary of the island for the purposes of computing area. Both the maximum flux $\psi$ and the total area $A$ remained nearly constant throughout the simulation, with variations having standard deviations of $2 \%$ and $7 \%$ of the mean, respectively. These merging rules reveal why the merging 


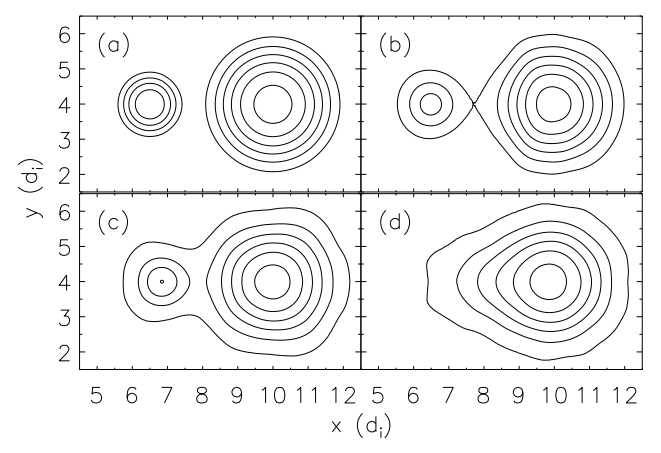

FIG. 1: The results of a PIC simulation of islands merger, showing the magnetic field lines at (a) $t=0 \Omega_{c i}^{-1}$, (b) $t=$ $0.3 \Omega_{c i}^{-1}$, (c) $t=4.0 \Omega_{c i}^{-1}$, and (d) $t=8.0 \Omega_{c i}^{-1}$, where $\Omega_{c i}$ is the ion cyclotron frequency. The smaller island initially has $75 \%$ of the flux and $25 \%$ of the area of the larger island.

process is energetically favorable: the dissipation of magnetic energy in the reconnection process. We can write the magnetic energy in an island as $W=B^{2} A \sim \psi^{2}$. Before the merger the energy is given by $W_{i} \sim \psi_{1}^{2}+\psi_{2}^{2}$, and after the merger the energy (supposing $\psi_{1} \leq \psi_{2}$ without loss of generality) is $W_{f} \sim \psi_{2}^{2}<W_{i}$.

We now develop an equation for the island distribution $f$ that parallels the derivation of the collisional Boltzmann equation. In the absence of island merging the number density of magnetic islands is preserved as reconnection changes an island's area and flux, while merging (analgous to collisional scattering in the case of the Boltzmann equation) causes a jump in the local island number. We first write the merging velocity of two islands with fluxes $\psi_{1}, \psi_{2}$ and areas $A_{1}, A_{2}$, assuming constant mass density $\rho$,

$v^{2}\left(\psi_{1}, A_{1}, \psi_{2}, A_{2}\right)=\frac{\varepsilon^{2}}{4 \pi \rho} \frac{\psi_{1} \psi_{2} r_{1} r_{2}\left(r_{1}^{2}+d_{i}^{2}\right)^{1 / 2}\left(r_{2}^{2}+d_{i}^{2}\right)^{1 / 2}}{\left(r_{1}^{2}+d_{e}^{2}\right)^{3 / 2}\left(r_{2}^{2}+d_{e}^{2}\right)^{3 / 2}}$

where the dependence on $A_{1}$ and $A_{2}$ is implicit in $r_{1}$ and $r_{2}$. For islands larger than $d_{i}$, this formula resembles the hybrid Alfvén velocity for asymmetric reconnection, $v^{2}=B_{1} B_{2} / 4 \pi \rho[10,11$. For smaller islands, down to the electron skin depth $d_{e}, v^{2} \approx \psi_{1} \psi_{2} d_{i}^{2} / 4 \pi \rho r_{1}^{2} r_{2}^{2}$ in agreement with the dispersion relation $v_{p h} \propto k$ of the whistler dynamics that dominate this regime. Lastly, $v \rightarrow 0$ as $r \rightarrow 0$.

Now we consider the number of islands $\left.\Delta N\right|_{\text {src }}(\psi, A)$ formed by merging. A merged island with flux $\psi$ arises from an island of flux $\psi$ that has merged with another island with flux $\psi^{\prime} \leq \psi$. Likewise, an island with area $A$ must come from an island with $A^{\prime}<A$ that has merged with an island of area $A-A^{\prime}$. The probability of those two islands merging within a time $\Delta t$ is given by $v\left(\psi, A^{\prime}, \psi^{\prime}, A-A^{\prime}\right) \Delta t / L$, so

$\left.\Delta N\right|_{\mathrm{src}}=\int_{0}^{A} d A^{\prime} f\left(\psi, A^{\prime}\right) \int_{0}^{\psi} d \psi^{\prime} \frac{v \Delta t}{L} f\left(\psi^{\prime}, A-A^{\prime}\right) \Delta \psi \Delta A$.

A similar analysis yields the number of islands lost through merging $\left.\Delta N\right|_{\mathrm{snk}}(\psi, A)$. Such an island is lost if it merges with any island of finite flux $\psi^{\prime}$ and area $A^{\prime}$. Again, the probability of merger depends on $v\left(\psi, A, \psi^{\prime}, A^{\prime}\right) \Delta t / L$, and

$\left.\Delta N\right|_{\mathrm{snk}}=-\int_{0}^{\infty} d A^{\prime} \int_{0}^{\infty} d \psi^{\prime} \frac{v \Delta t}{L} f\left(\psi^{\prime}, A^{\prime}\right) f(\psi, A) \Delta \psi \Delta A$.

The rate of change of $f$ due to merging is then calculated as $\left(\left.\Delta N\right|_{\operatorname{src}}+\left.\Delta N\right|_{\mathrm{src}}\right) / \Delta \psi \Delta A \Delta t$. Combining this expression with the change in $f$ due to reconnection, we get our evolution equation:

$$
\begin{aligned}
& \frac{\partial f}{\partial t}+\frac{\partial}{\partial \psi}(\dot{\psi} f)+\frac{\partial}{\partial A}(\dot{A} f)=S(\psi, A)-\frac{c_{A}}{L} f \\
& +\frac{1}{L} \int_{0}^{A} d A^{\prime} f\left(\psi, A^{\prime}\right) \int_{0}^{\psi} d \psi^{\prime} v\left(\psi, A^{\prime}, \psi^{\prime}, A-A^{\prime}\right) f\left(\psi^{\prime}, A-A^{\prime}\right) \\
& -\frac{1}{L} f(\psi, A) \int_{0}^{\infty} d A^{\prime} \int_{0}^{\infty} d \psi^{\prime} v\left(\psi, A, \psi^{\prime}, A^{\prime}\right) f\left(\psi^{\prime}, A^{\prime}\right) .
\end{aligned}
$$

On the left-hand side, the time-derivative of $f$ results from the change in flux $\dot{\psi}$ and area $\dot{A}$ due to reconnection, which act to increase the island size and therefore conserve total island number. On the right-hand side, we have a source $S(\psi, A)$, a sink representing island convection out of the system, and the merging terms. Consistent with the merging rules, the merging terms in Eq. (4) preserve total area, which can be shown by multiplying by $A$ and integrating over $A$ and $\psi$.

We first consider the case where the merging terms are negligible. This is valid if there are few islands in the layer $(S(\psi, A)$ is small). For simplicity, we change variables from the area $A$ to a characteristic radius $r$, with $A=\pi r^{2}$ (although the islands need not be circular) and the new distribution function,

$$
F(\psi, r)=f(\psi, A) \frac{d A}{d r}=2 \pi r f\left(\psi, \pi r^{2}\right) .
$$

The evolution equation, without merging, becomes

$$
\frac{\partial F}{\partial t}+\frac{\partial}{\partial \psi}(\dot{\psi} F)+\frac{\partial}{\partial r}(\dot{r} F)=S(\psi, r)-\frac{c_{A}}{L} F .
$$

With a delta function source $S=\delta\left(\psi-\psi^{\prime}\right) \delta\left(r-r^{\prime}\right)$, the steady-state solution $F_{G}$ is given by the Green's function

$$
\begin{aligned}
F_{G}\left(\psi, r, \psi^{\prime}, r^{\prime}\right)= & \frac{1}{\varepsilon c_{A}} \exp \left(-\frac{r-r^{\prime}}{\varepsilon L}\right) H\left(r-r^{\prime}\right) \\
& \times \delta\left(\left(\psi-\psi^{\prime}\right)-B_{0}\left(r-r^{\prime}\right)\right)
\end{aligned}
$$

where $H(r)$ is the Heaviside function. All islands in this solution have a magnetic field strength $B_{0}$, equal to the 


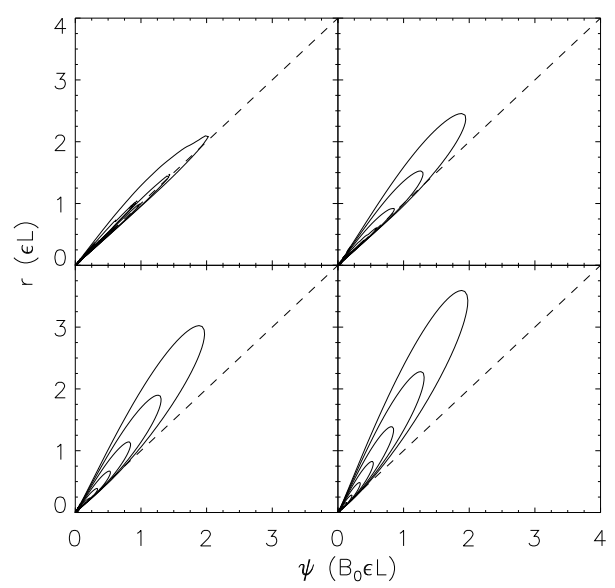

FIG. 2: The steady-state distribution function $F_{\infty}(\psi, r)$ for (a) $S_{N}{ }^{*}=4$, (b) $S_{N}{ }^{*}=40$, (c) $S_{N}{ }^{*}=400$, and (d) $S_{N}{ }^{*}=4000$. The contours are logarithmic.

ambient magnetic field. The solution in $\psi-r$ phase space is a decaying exponential along the line $\psi=B_{0} r$, starting at $\psi=\psi^{\prime}$ and $r=r^{\prime}$. The corresponding solutions for $f$ are shown in dashed lines in Fig. 2, A key feature of Eq. (7) is the characteristic island size $r_{0}=\varepsilon L$, which arises from balancing the island growth rate of $\dot{r}=\varepsilon c_{A}$ with a system transit time of $L / c_{A}$. For example, with a reconnection rate of $\varepsilon \sim 0.1$ on the magnetopause where $L \sim 30 R_{E}$, this simple model predicts islands of size $\sim 3 R_{E}$. A survey of flux transfer events along the magnetopause [12, 13] determined that typical scale sizes are $0.5 R_{E} \times 2 R_{E}$, for which $r \approx 1 R_{E}$. Such island sizes are also consistent with those seen in direct observations of current sheets formed by CMEs [14].

If the merging terms are kept, the resulting integrodifferential equation must be solved computationally. Although Eq. (4) appears to contain several parameters (the current sheet length $L$, the reconnection rate $\varepsilon$, and the source term strength $S_{N}=\int_{0}^{\infty} d r \int_{0}^{\infty} d \psi S(\psi, r)$ which gives the rate of island nucleation), in reality it only has one free parameter. By normalizing time to $L / c_{A}$, island flux to $\varepsilon B_{0} L$, island size to $\varepsilon L$, and the merging velocity to $\varepsilon c_{A}$, the only free parameter is the normalized amplitude of the source, $S_{N}{ }^{*}=S_{N} \varepsilon L / c_{A}$. Although we set $L=4000 d_{i}$ here (a realistic value for the magnetopause), we can essentially model any system by varying $S_{N}$.

The integration of Eq. (4) yields the steady state island distribution, $F_{\infty}(\psi, r)$, which is shown for four values of $S_{N}$ in Fig. 2. Whereas the solution without merging remains localized along the $\psi=B_{0} r$ diagonal, the merging terms break this symmetry: the merging process increases area but not flux so the distribution functions curve away from the diagonal toward larger $r$. One test of the veracity of our numerical solutions is their consistency with the moments of the evolution equation. In- tegrating Eq. (4) in $\psi$ and $A$ yields an equation for the total number of islands $N=\int_{0}^{\infty} d A \int_{0}^{\infty} d \psi f(\psi, A, t)$ :

$$
\frac{d N}{d t}=S_{N}-\frac{c_{A}}{L} N-\frac{\varepsilon c_{A}}{2 L} N^{2}
$$

where, for simplicity, we have taken the merging velocity to be $\varepsilon c_{A}$. The merging terms reduce $N$ since two islands merge into one and are responsible for the term quadratic in $N$. Eq. (8) can be solved analytically:

$$
N(t)=\frac{N_{f}\left(1-e^{-t / t_{s}}\right)}{1+\left(\varepsilon^{2} N_{f}^{2} / 2 S_{N}^{*}\right) e^{-t / t_{s}}}
$$

where

$$
N_{f}=\frac{1}{\varepsilon}\left[\left(1+2 S_{N}^{*}\right)^{\frac{1}{2}}-1\right], t_{s}=\frac{L}{c_{A}}\left(1+2 S_{N}^{*}\right)^{-\frac{1}{2}}
$$

are the asymptotic number of islands in steady state and a time-scale to reach it. The form of $N(t)$ predicted by Eq. (9) compares favorably to that of the numerical solution. Note from Eq. (10) that $S_{N}$ dictates the number of islands in the system, and so it effectively controls the importance of merging. For larger $S_{N}$, the merging term $\left(\sim N^{2}\right)$ in Eq. (8) dwarfs the convective loss term $(\sim N)$.

Similarly, by multiplying Eq. (4) by $A$ and integrating, we get a moment equation for the total area of all the islands in the system, $A_{T}=\int_{0}^{\infty} d A \int_{0}^{\infty} d \psi A f(\psi, A, t)$ :

$$
\frac{d A_{T}}{d t}=2 \pi \varepsilon c_{A} r_{T}-\frac{c_{A}}{L} A_{T}
$$

where $r_{T}=\int_{0}^{\infty} d r \int_{0}^{\infty} d \psi r F(\psi, r, t)$ and the (small) source contribution was neglected. Since merging conserves total area, the merging integrals do not contribute to this equation.

Fig. 3(a) shows the distribution of islands in radius,

$$
\bar{F}_{\infty}(r)=\int_{0}^{\infty} d \psi F_{\infty}(\psi, r)
$$

for each $S_{N}$. The behavior of these curves for the largest islands can be deduced from Eq. (4) for large $A$. Integrating over $\psi$, assuming a constant merging velocity $v=\varepsilon c_{A}$, and expanding the integral over the merging terms gives an equation for $\bar{f}(A, t)=\int_{0}^{\infty} d \psi f(\psi, A, t)$ :

$$
\frac{\partial \bar{f}}{\partial t}+\frac{\partial}{\partial A}(\dot{A} \bar{f})+\frac{\varepsilon c_{A} A_{T}}{L} \frac{\partial \bar{f}}{\partial A}=-\frac{c_{A}}{L} \bar{f}
$$

The third term on the left side, which arises from the merging terms, describes how large islands grow in area by devouring smaller islands - the coefficient $\varepsilon c_{A} A_{T} / L$ is the rate at which the total area $A_{T}$ of all the smaller islands is consumed. By balancing the second and third terms on the left side we obtain a characteristic length scale above which growth via reconnection dominates growth via merging. Recalling that $\dot{A}=2 \pi \varepsilon c_{A} r$, we find that the transition occurs at $\hat{r}=A_{T} / 2 \pi L$. In steady state Eq. (11) yields $A_{T}=2 \pi \varepsilon L r_{T}$ so $\hat{r}=\varepsilon r_{T}$. 


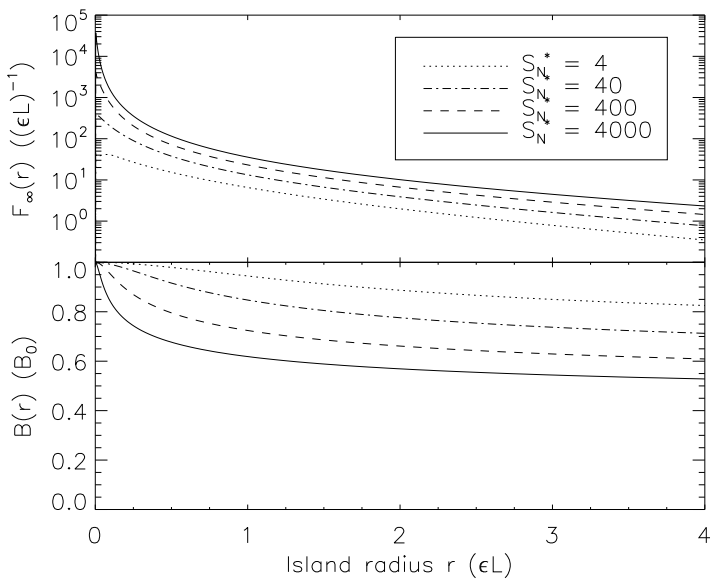

FIG. 3: (a) The steady-state distribution function in $r$, given by $F_{\infty}(r)$ as defined in Eq. (12) for various $S_{N}$. (b) The average magnetic field strength $B$ as a function of island radius $r$ for various $S_{N}$.

Equation (13) admits a steady state solution,

$$
\bar{F}_{\infty}(r)=C e^{-r / \varepsilon L} r\left(r+\varepsilon r_{T}\right)^{r_{T} / L-1},
$$

where $C$ is an arbitrary constant. For sufficiently large $r$, the exponential behavior of Eq. (14) will dominate. In all four cases shown in Fig. 3 the distribution of islands at large radii agrees with the expression given in Eq. (14).

Merging increases an island's area but not its flux, and hence leads to a decreased in-plane field strength $B=$ $\psi / r$. Since merging scales as $N^{2}$, one might surmise that the more islands in the system (e.g., because of larger
$S_{N}$ ), the more merging takes place, and the smaller the magnetic field $B$. This hypothesis is borne out in Fig. 2, in that the steady-state solution for larger $S_{N}$ tilts farther away from the $\psi=B_{0} r$ diagonal. This effect is seen in Fig. 3(b), which shows, for various $S_{N}$, the average magnetic field strength as a function of island radius

$$
B(r)=\frac{\int_{0}^{\infty} d \psi \frac{\psi}{r} F_{\infty}(\psi, r)}{\int_{0}^{\infty} d \psi F_{\infty}(\psi, r)} .
$$

Comparisons of the present results with those of recent MHD simulations of large current layers [15] are not possible since these simulations were limited to the early time behavior, where island merging plays a minimal role. Therefore, in future work we will benchmark this model using Hall MHD simulations to justify our equations for a current layer with many islands. Although observational data (for example, from THEMIS at the magnetopause) cannot directly measure $S_{N}$, the known distributions of island sizes and magnetic field strengths could, using our model, be used to infer it. An encouraging early result is a histogram of 1223 FTEs detected by THEMIS between 2007 and 2008, which shows an exponential decrease in event number for island scale lengths above an $R_{E}$ [16]. Similarly, TRACE observations of supra-arcade downflows in the solar corona during flares could also yield distributions of island size [17.

This work was supported by NASA through an Earth and Space Science Fellowship NNX07A083H and NNX09A102G. Computations were performed at the National Energy Research Scientific Computing Center.
[1] J. F. Drake, M. Swisdak, K. M. Schoeffler, B. N. Rogers, and S. Kobayashi, Geophys. Res. Lett. 33, L13105 (2006).

[2] C. T. Russell and R. C. Elphic, Geophys. Res. Lett. 6, 33 (1979).

[3] N. R. Sheeley, H. P. Warren, and Y.-M. Wang, Astrophys. J. 616, 1224 (2004).

[4] A. Ciaravella and J. C. Raymond, Ap. J. 686, 1372 (2008).

[5] J. F. Drake, H. Che, M. A. Shay, and M. Swisdak, Nature 443, 553 (2006).

[6] L.-J. Chen, A. Bhattacharjee, P. A. Puhl-Quinn, H. Yang, N. Bessho, S. Imada, S. Muhlbachler, P. W. Daly, B. Lefebvre, Y. Khotyaintsev, A. Vaivads, A. Fazakerley, and E. Georgescu, Nature Phys. 4, 19 (2008).

[7] W. Daughton, V. Roytershteyn, B. J. Albright, H. Karimabadi, L. Yin, and K. J. Bowers, Phys. Rev. Lett. 103, 065004 (2009).

[8] J. Raeder, Annales Geophysicae 24, 381 (2006).
[9] M. A. Shay, J. F. Drake, and M. Swisdak, Phys. Rev. Lett. 99, 155002 (2007).

[10] P. Cassak and M. A. Shay, Phys. Plasmas, 102114(2007).

[11] M. Swisdak and J. F. Drake, Geophys. Res. Lett. 34, L11106 (2007).

[12] R. P. Rijnbeek, S. W. H. Cowley, D. J. Southwood, and C. T. Russell, J. Geophys. Res. 89, 786 (1984).

[13] M. A. Saunders, C. T. Russell, and N. Sckopke, Geophys. Res. Lett. 11, 131 (1984).

[14] J. Lin, Y.-K. Ko, L. Sui, J. C. Raymond, G. A. Stenborg, Y. Jiang, S. Zhao, and S. Mancuso, Ap. J. 622, 1251 (2005).

[15] R. Samtaney, N. F. Loureiro, D. A. Uzdensky, A. A. Schekochihin, and S. C. Cowley, Phys. Rev. Lett. 103, 105004 (2009).

[16] H. Zhang(2009), private communication.

[17] D. E. McKenzie and S. L. Savage, Astrophys. J. 697, 1569 (2009). 